\title{
To design is to design oneself
}

Silvano Tagliagambe

\begin{abstract}
This article begins with the analogy between work in philosophy and work in architecture proposed by Wittgenstein, based on the idea that in both cases we are faced with work on ourselves and on the construction of our own identity and general understanding.

This initial idea is developed by explaining how all project-based concern requires first and foremost the raising of one's internal resources to a level of competence and knowledge that puts the subject in a position to tangibly handle the problems the project entails.

On the grounds of further conviction that the project should always be nurtured both on concern for change/ inclination towards seeing and thinking differently, therefore "sense of possibility", and on capacity for rooting, namely sense of reality, reference is made to the "Triadic System of Rooting and Projection (TSRP)". This is a perceptive and cognitive nucleus guaranteed by three different processing systems: ecological intelligence (the motor-perceptive system and devices linked with the representation of space); social intelligence (the mind's system of reading applied to the construction of a space shared with other organisms); finally, temporal intelligence (the capacity to travel in time on which the construction of the experiential continuity of individuals is based). Though they process very different types of information, these three cognitive systems converge on the capacity to detach the organism from the current situation and project it into alternative situations in time, space and the social environment.

Following a specific analysis of the first two systems, we dwell on the different components of temporal intelligence, proposing a new interpretation of the articulate concept of time we inherited from Greek culture, with the purpose of understanding which idea of time is the most suitable for us to understand the speed of innovation nowadays and thus to support project-oriented culture in line with the requirements of our times.

The article ends with a detailed analysis of the three stages that characterise visual perception, as proposed by the results neuroscience has achieved, with the aim of deepening the contribution that deduction, induction and abduction, as unique "instruments for thinking", must provide for project-oriented work.
\end{abstract}

"As is frequently the case with work in architecture, work on philosophy is actually closer to working on oneself. On one's own understanding. On the way one sees things. (And on what one demands of them.)" L. Wittgenstein, The Big Typescript: TS (Wittgenstein, 2002).

213 German-English Scholars' Edition, edited and translated by C. Grant Luckhardt and Maximilian A.E. Aue, Wiley-Blackwell (2005), Blackwell Publishing Ltd., Oxford, UK.

\section{The monkey and perseus}

The greatest barrier to concern over design and our ability to process it is the way we see, what we might call

Correspondence: sil.tagliagambe@gmail.com

Department of Architecture and Planning, Faculty of Architecture, University of Sassari, Palazzo del Pou Salit, Piazza Duomo 6, 07041 Alghero, Italy our "gaze strategy", which profoundly affects our way of perceiving and experiencing the environment we live in. This strategy is often the product of a unilateral type of sight and of bias that works like the old Indian monkeytrap Pirsig spoke of: "The trap consists of a coconut shell chained to a fence. It contains rice that can be taken through a hole. The opening is big enough for a monkey to get its hand in, but too small for it to get it out full of rice. The monkey puts its hand in and finds it trapped - solely due to the inflexibility of its values. It cannot change the value of the rice. It cannot manage to see that freedom without rice is worth more than capture with it" (Pirsig, 1981, p. 301).

What acts as "rice" in the case in point is a vision of the future unfit to accommodate the deep sense of change we are experiencing and the radical innovations

\section{空}

(c) 2014 Tagliagambe; licensee Springer. This is an Open Access article distributed under the terms of the Creative Commons Attribution License (http://creativecommons.org/licenses/by/4.0), which permits unrestricted use, distribution, and reproduction in any medium, provided the original work is properly credited. 
characterising our times. This "trap" therefore concerns the fundamental issue of time and our relationship with it and is the fruit of our inability to realise that, today more than ever, the past may certainly provide us with instructive, emblematic cases but not answers and effective remedies since exact repeatability just does not exist. This theme is at the heart of a challenging book by Nassim Nicholas Taleb, entitled, not by chance, The Black Swan. The Impact of the Highly Improbable, in which the author poses the problem of the growing difficulty of imagining a future that has not been extrapolated from the present, a projection of today on tomorrow, which we consider - precisely because it is thought of in this way - can be coped with by referring back to the same prevalent, hegemonic conceptual pictures of the moment (Taleb 2008). If we follow this path we will actually only achieve "learning backwards", accepting that we can and should do without any reference to the unusual and the "unpredictable" for they "cannot be extrapolated" and are therefore "not normal" by definition.

Those who follow an approach like this are not able to imagine a future without an "algorithmic" face - the result of a regular succession and spontaneous projection of the "before" on the "after" - whereas it should be conceived as "life to come", loaded with unpredictability so full of risks, but also of hopes and opportunities.

The idea of a "project-oriented" person should be contrasted with Pirsig's monkey, a person convinced that it is up to man's action to proactively and effectively "weave" the various threads that make up his existence in the world, and that man should therefore constantly be committed to producing a constructive capacity that will allow him to tackle the challenges of the present and near future in ways and shapes that match up to what is at stake.

This type of person is portrayed well by Perseus, the mythological hero of whom Italo Calvino gives us a powerful description in an essay belonging to the American Lessons he would have given at Harvard in the 1985/86 academic year, if he had not suffered a stroke in September 1985 on the eve of his departure for the United States (Calvino, 2002).

In the first of these lessons, devoted to Lightness, the author reflects on the meaning of Medusa's inexorable stare that turns every aspect of life to stone - and can therefore be considered an effective representation of the inertia of reality and its resistance to every effort and attempt to change it - and on the way of getting round it and fighting it:

"The only hero able to cut off Medusa's head is Perseus, who flies with winged sandals; Perseus, who does not turn his gaze upon the face of the Gorgon but only upon her image reflected in his bronze shield. [...] To cut off Medusa's head without being turned to stone, Perseus supports himself on the very lightest of things, the winds and the clouds, and fixes his gaze upon what can be revealed only by indirect vision, an image caught in a mirror" (Six Memos for the Next Millennium, 2008, p. 12).

So in order to oppose the petrification process of reality we need to make use of the resources of lightness and at the same time benefit from the advantage of "indirect vision", which enables us to look at what surrounds us through a mirror image. This dual reference to lightness and the mirror image has a clear-cut meaning. Aristotle adopts lightness both in his Physics and De Anima as a principle of movement in natural bodies, in that it is form and nature, namely formal cause and nature (Aristotele, 2012). To form, living" form in particular, is attributed motor causality, a certain type of

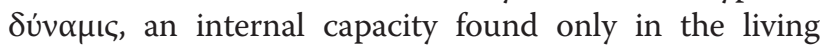
and specifically in man, in whom the soul is both form and at the same time principle of movement.

As far as the mirror is concerned, apart from creating the imaginary, Lacan considers it the fundamental instrument of the identification process. The expression "mirror stage" is in fact used in evolutionary psychology with a Lacanian matrix to indicate the cognitive process within the range of six to eighteen months of age in which the child manages to recognise the image he sees in the mirror as his own. If placed in front of a mirror, a child first reacts with a sense of alienation, as if he were able to interact with the image he sees; only later will he realise the imaginary status of that vision. Finally, he will reach the point of understanding that what he sees is not just an image but his own image (Lacan, 1974, pp. 89 et seq.).

So it is through the image the mirror yields that, in an initial phase, the moment of transformation of the subject takes place; thanks indeed to the image identifying him, in which he recognises himself and what belongs to him, distinguishing himself from the surrounding world, the subject becomes aware of his own reality and nature.

The dual reference to lightness and the specular image in the myth of Perseus therefore means that Medusa, hence heaviness and resistance to reality, may successfully be fought only by filtering a solid self-awareness. A prerequisite of the capacity to intervene in the environment in which one lives and modify it is therefore the enhancement of the self as a person, the raising of one's internal resources to a level of competence and knowledge that, as well as giving solid awareness, puts the subject in a position to tangibly handle the problems each project entails.

Perseus's strength, Calvino continues, and his struggle against Medusa do not, however, turn into "a refusal of the reality in which he is fated to live; he carries the reality with him..." (Calvino, 2012).

In fact the myth tells us that:

"The relationship between Perseus and the Gorgon is a complex one and does not end with the beheading of 
the monster. Medusa's blood gives birth to a winged horse, Pegasus - the heaviness of stone is transformed into its opposite. [...] As for the severed head, Perseus does not abandon it but carries it concealed in a bag. When his enemies are about to overcome him, he has only to display it, holding it by its snaky locks, and this bloodstained booty becomes an invincible weapon in the hero's hand. It is a weapon he uses only in cases of dire necessity, and only against those who deserve the punishment of being turned into statues. Here, certainly, the myth is telling us something, something implicit in the images that can't be explained in any other way. Perseus succeeds in mastering that horrendous face by keeping it hidden, just as in the first place he vanquished it by viewing it in a mirror. Perseus's strength always lies in a refusal to look directly, but not in a refusal of the reality in which he is fated to live; he carries the reality with him and accepts it as his particular burden" (Six Memos for the Next Millennium, 2008, p. 12-13).

When read in this key the myth tells us however that design has to be nurtured both on concern for change/inclination towards seeing and thinking differently, therefore "sense of possibility", and on capacity for rooting, namely sense of reality. To understand how this co-existence may tangibly be created, reference is made to the process of "contextually bound flexibility", the mechanism of which we are nowadays beginning to understand fully, also from the biological and evolutionary points of view. It involves two basic abilities usually shown in intelligent behaviour: the capacity to "anchor" oneself to the context (the function that strongly roots the organism in the contextual situation) and the capacity to "project" oneself from the current context into a different one (the function able to detach or dissociate the organism from the here and now of the present situation). Rooting and projection constitute the basic functions of flexibly adequate behaviour and therefore also of successful design.

Let us revert to Calvino, who continues as follows:

"On the relationship between Perseus and Medusa, we can learn something more from Ovid's Metamorphoses (Calvino, 2012). Perseus wins another battle: he hacks a sea-monster to pieces with his sword and sets Andromeda free. Now he prepares to do what any of us would do after such an awful chore - he wants to wash his hands. But another problem arises: where to put Medusa's head. And here Ovid has some lines (IV. 740-752) that seem to me extraordinary in showing how much delicacy of spirit a man must have to be a Perseus, the killer of monsters: "So that the rough sand should not harm the snake-haired head, he makes the ground soft with a bed of leaves, and on top of that he strews little branches of plants born under water, and on this he places Medusa's head, face down". I think that the lightness, of which Perseus is the hero, could not be better represented than by this gesture of refreshing courtesy toward a being so monstrous and terrifying yet at the same time somehow fragile and perishable. But the most unexpected thing is the miracle that follows: when they touch Medusa, the little marine plants turn to coral and the nymphs, in order to have coral for adornments, rush to bring sprigs and seaweed to the terrible head" (Six Memos for the Next Millennium, 2008, p. 13).

Now, the respect for reality and "kindness" towards it, even when our desire for change makes it revocable, is nevertheless fragile; not only is it not a hindrance to transformation and change but on the contrary constitutes the indispensable prerequisite.

This necessary complementarity between sense of reality and sense of possibility is assured by what is called the "Triadic System of Rooting and Projection" (TSRP), in its turn "guaranteed by three different processing systems: ecological intelligence (the motor-perceptive system and devices linked with the representation of space); social intelligence (the mind's system of reading applied to the construction of a space shared with other organisms); finally, temporal intelligence (the capacity to travel in time on which the construction of the experiential continuity of individuals is based" (Ferretti, 2009, p. 87).

Though they process very different types of information, the three cognitive systems in question converge on the capacity to detach the organism from the current situation and project it into alternative situations in space, time and the social environment. This convergence is witnessed by the joint operation of the subcomponents involved in various cognitive tasks: the capacity to represent space is very often linked with the capacity to represent time; the capacity to "look at the world with the eyes of others" also necessarily entails spatial dislocation.

Given their effects on project-oriented capacity, it is worth analysing these three cognitive systems in depth and separately.

\section{Ecological intelligence}

The findings of research on the structure and functioning of some cerebral processes and circuits are increasingly eroding away the credibility of the classical pattern of perception $\Rightarrow \operatorname{cognition} \Rightarrow$ movement, which has now entered an irresolvable crisis due to recognition of the fact that perception appears to be immersed in the dynamics of action and consequently proves much more articulate and composite than it was shown to be in the past. This conclusion was reached thanks to research highlighting that the motor system is by no means peripheral and isolated from the rest of cerebral activity. On the contrary, it consists of a complex weft of areas differentiated in terms of location and functions, and able to provide a decisive contribution to achieving 
those motor-sensory transformations on which the individuation/location of objects and activation of movements depend that are required by most of the acts and behaviour comprising our daily experience. "And this is not all: the fact that sensory and motor information can be traced back to a common format, coded by specific parieto-frontal circuits, suggests that as well as organising our motor behaviour, certain processes usually considered of a higher order and attributed to systems of a cognitive type, such as, for example, the perception and recognition of others' acts, imitation and even forms of communication by gestures and voice, may also refer back to the motor system and find their primary neural substrate in it" (Rizzolatti and Sinigaglia 2006, p. 22).

In this extremely interesting general picture are included the results achieved by a research group at the University of Parma under Giacomo Rizzolatti, an avant-garde scholar in the experimental neurophysiology sector, which led to the discovery of "mirror neurons" - so-called since they permit a form of mirroring between perception and action - in the F5 area of the ventral premotor cortex of the macaque monkey, considered homologous with the Broca area in humans, meaning that it may be interpreted as an evolution of the F5 area of monkeys.

This discovery strengthens the idea that the recognition of objects is entrusted to a "pragmatic" representation (the fact that an object can be grasped in a certain way) rather than a semantic representation of reality. From this viewpoint, therefore, seeing occurs that is not an end in itself but directed towards steering the hand, and consequently presents itself "also, if not above all, as seeing with the hand, with respect to which the object perceived appears immediately to be coded as a specific set of hypotheses for action" (Rizzolatti and Sinigaglia 2006, p. 49).

Perception, therefore, reveals itself as an implicit preparation of the body to respond and act, and a type of understanding springs from it whose nature is in fact eminently pragmatic and does not in itself determine any "semantic" representation of the object. Rather than being, for example, identified and recognised on the grounds of its form, meaning or function, in a word its belonging to an already classified, coded set, the said object would therefore be perceived and recognised simply as something that can be grasped by the hand. "F5 and AIP neurons only respond to certain features of objects (their shape, size, inclination, etc.), and this selectivity is significant in that these features are interpreted as just as many systems of visual affordances and potential motor acts. Whereas the neurons populating the lower cerebral cortex areas code profiles, colours and wefts of objects, processing the information selected in images that, once memorised, would enable them to be recognised in their visual features. But is this enough to resolve the anatomical distinction between the ventral and dorsal routes in the functional opposition between vision-for-perception and vision-for-action? We do not think so - unless perception is reduced to an iconic representation of objects, the portrayal of a thing, independent from any where or how, and action reduced to an intention that discriminates between how and perhaps where, but has nothing to do with what. Unless, that is, the perceptive process is relegated to a mere identification of figures (ideas, in the literal sense of the word), corrected of any motor meaningfulness and raised to the rank of single possible vehicles of meaning, and the sense of the action broken up into a simple succession of movements in themselves devoid of any objective correlative" (Rizzolatti and Sinigaglia 2006, p. 49-50).

For the practical opportunities it enables this hypothesis, on the basis of which any object proves knowable and can therefore be conceptualised, highlights, on the one hand, the inseparable link between perception and action, and emphasises, on the other, how the act of perceiving is not the result of a merely contemplative attitude towards reality but the expression of an activity in which the subject takes root in the environment by constantly transforming it. This means placing design at the base of perception, as its initial prerequisite, and not as its final result, with a merely applicative, executive nature.

This conclusion is accredited and reinforced further by the growing awareness that nowadays, as has rightly been pointed out (Postman 1983), the reality we live in is more and more marked by an exponential increase in information and knowledge to the point of making us all somewhat victims of a sort of cultural AIDS (Anti-Information Deficiency Syndrome), due to the growing weakness of any type of immune system against information. The social and cultural structures which once acted as powerful filters of the latter, selecting it, are gradually losing their strength and effect: religion (in the sacred text there is everything it is essential to know); the family (with the tendency of parents to choose the communicative and expressive forms of their children, imposing certain books and themes of conversation and forbidding others); school (which constitutes the cultural tradition, gives it shape, perpetuates it and conveys the contents that each social system considers fundamental); science (which, through the creation of schools, spreading of manuals, and due to the effects of what Kuhn has called "paradigms" on it, tends in each phase of its development to provide a model of problems and solutions acceptable by all those working in a certain field of research), and so on. The result is the chaos of information that nowadays is oppressing and suffocating us from all sides and within which it is more and more difficult to find rules and a direction. 
Thanks to the microelectronic revolution, conveying and recording information has become more and more simple and inexpensive. The enormous exchange of data and the multiplication of active and passive support devices (memories, networks, calculators, work stations and databanks) made possible by the progress in information and communication technologies offer an ever wider spectrum of new communicative, cultural and aggregative possibilities, with the formation of small and large communities linked in networks that join up and dissolve in the pursuit of a more or less lasting common interest.

This increasingly unconditional freedom of access to information that risks producing the dilution and loss of the sense of communication as an undesirable downside, requires a capacity to select the information and knowledge available, which needs to be based - in order to be carried out in a reasonable, effective and not purely casual, inappropriate way - on a precise criterion of pertinence. Since utterly pertinent/non pertinent cognitive data or information do not exist, however, if this selection is to be performed in the rigorous manner we have suggested (which ought to be expected), it requires preliminary reference to a problem to be tackled or project to be worked out. It is only in relation to such a problem/project, in actual fact, that we can reasonably establish which information and which knowledge is pertinent and useful and which not. If, therefore, for the reasons explained, perception is to be understood more and more as a selective capacity, it is solely through explicit reference to a problem or project that such a capacity may be tangibly and properly manifested.

The meaning of this change of scenario becomes clear if we refer to Gibson's idea that objects, even the simplest and most trivial, contain more than one affordance. In the case of a common coffee-cup the visual affordances offered to our motor system concern the handle, central body, upper rim, etc. Consequently, observing it "will determine the activation of several neural populations in the upper intraparietal area (UIA), each of which codes a specific affordance. It is probable that these "proposals" for action be sent to the F5 area, triggering authentic potential motor acts. Now the choice of how to act will not just depend on the intrinsic properties of the object in question (shape, size, inclination, etc.) but also on what we intend to do with it, and the use functions we acknowledge it has, etc. In the case of the cup, for example, we will grasp it in different ways depending on whether we want to pick it up to drink coffee, rinse it or, more simply, move it. And already in the first case our grip may be different according to whether we are afraid of being burnt, if there are any objects surrounding the cup, our habits, and whether we are inclined to respect good manners, etc." (Rizzolatti and Sinigaglia 2006, p. 36).
As regards the representation of space, which constitutes the other cornerstone of "ecological" intelligence, useful suggestions come to us from echographic findings of the foetus' behaviour in its mother's uterus. After the eighth week it already shows abundant targeted motor activity, e.g. it moves its hand towards its face, while at the sixth month it is able to put its hand in its mouth and suck it - which demonstrates that before the child is born it possesses motor representation of space. Once born, its movements become more and more targeted, based on the space surrounding its body, and this provides good evidence to support the idea, already put forward by Ernst Mach and Jules-Henri Poincaré, of the existence of a peripersonal space, coded in somatic coordinates, i.e. processed in relation to the body and its motor possibilities. As Poincaré writes, in fact, "it would have been impossible for us to build" something like a space "if we had not had an instrument for measuring it" an instrument to which we could "relate" each thing and that we could use "instinctively", namely our body: "It is in relation to our body that we situate external objects, and the only relations of these objects that we can represent to ourselves are their relations with our body" (Poincaré 1989, p. 217).

So, peripersonal space is defined by Poincaré in terms of the mutual coordination of the "many parries" made possible by simply extending the arm: it is this extension that enables us to determine the distinction between near (everything that comes within the radius of action outlined by it and therefore proves within range) and far (everything that is beyond this range), and thus between peripersonal and extrapersonal space. The distinction, however, "is not defined once and for all, for the space it marks out cannot be thought of as static but must be conceived in a dynamic form. In other words, the distinction between near and far cannot be reduced to a mere question of centimetres, as if our brain were calculating the distance that separates our body from objects in absolute terms only. All this would not just contradict that principle of relativity of space dear to Poincaré and decisive for the body's organisation of movements. The actual organisation of F4 neuron receptive fields and their anticipatory function with respect to contact with the skin is not compatible with the idea of a rigidly, unambiguously fixed peripersonal space". And, in fact, some experiments have shown how the visual receptive fields of bimodal neurons in the posterior parietal cortex of the monkey, which code hand movement in a similar way to F4 neurons, may be modified by actions that entail the use of instruments. After training some monkeys to retrieve pieces of food with a small fork, researchers "noted that during repeated use of the instrument the visual receptive fields anchored to the hand expanded to the point of including the internal space of the hand and 
the fork - almost as though the image of the latter was incorporated in that of the hand. Whereas, when the animal stopped using the instrument, though it still held it in its hand, the receptive fields returned to their usual range. The extension of the hand determined by the use of the fork entailed widening of the space reachable by the monkey, and therefore remodulation of near and far: the neurons that became active in the presence of objects in the peripersonal space also responded to stimuli that they had not coded previously for they were far (namely outside their space), but then, with the use of the fork, became near" (Rizzolatti and Sinigaglia 2006, p. 71).

We may therefore say that if, in the light of the results of the research we have referred to, objects appear more and more like poles of virtual acts, space is defined by the system of relations that these acts unfold, and which finds its own proportion in the various parts of the body. What we might call an ontology of relations thus takes on a precise outline, within which the places of space cannot be understood as positions having an autonomous meaning of their own, nor conceived as "objective positions" in relation to a likewise presumed objective position of our body, but need to be understood, as Merleau-Ponty taught us, in their "inscribing around us the variable range of our intentions or gestures" (Merleau-Ponty, 1965, p. 199). The reference to intentions highlights how we cognitively manage the world surrounding us with mental operations, so that the elements of the real are for us no longer "things" but, as has been seen, poles of possible operations that we can carry out - correlated with possible interactions between ourselves (our body) and the world. To interpret the world in this key is to prefigure (almost pre-glimpse) all the projects for action we could undertake on it, and know how to combine, therefore, as we have said, sense of reality and sense of possibility, managing to establish that harmonic balance between these two senses which Musil speaks of in Man without qualities:

"If the sense of reality exists, and no-one can doubt that its existence is justified, then there must also be something that we will call sense of possibility. Those who have it do not say, for example: here this or that happened, will happen, must happen, but imagine: here this or that could or should happen. And if you tell them something is what it is, they think: well, it could probably also be different. So that the sense of possibility could also be defined as the capacity to imagine everything that it could be, and not give greater importance to what it is than to what it is not" (Musil, 1957, $\mathrm{N}^{\circ} 12$ ).

Seen from this viewpoint, the nature of design, however it is intended and defined and whatever process is applied to it, becomes the expression of a continuous wager that must take into account, obviously, the ties placed by reality, but also remain open to a spectrum of possibilities with which to play, thus avoiding falling into the trap of one-sided exaltation of the ties to the detriment of the system of opportunities that should remain available once these have been defined and fixed.

\section{Social intelligence}

Another interesting aspect that has already arisen from the discovery of mirror neurons is the possible reference to a capacity based on precise neuron mechanisms in an observer, to "translate" on the spot the body perspective of a person observed carrying out a particular action. This means that to bring about this translation we have absolutely no need of "dictionary" mediation consisting of mental pictures, as classical cognitivism suggested, conceiving the mind as a functional system whose processes can be described as manipulations of information symbols based on a series of formal syntax rules. It was from this approach that the idea consequently sprang that pictures are intrinsically symbolic and that thought should be reduced to a merely computational process.

To thoroughly clarify the difference between the two approaches an example might be useful. If I see someone in a bar move their hand towards a tankard of beer, I will immediately understand that he is about to sip the drink. The crucial point is: how do I do it? According to the classical cognitive approach, to reach this conclusion I have to necessarily translate the sensory information relating to the gesture of the person I am observing into a series of mental pictures sharing the same prepositional format with language: in the case in point, these pictures will concern the other person's desire to drink beer, his beliefs concerning the fact that the tankard he is about to grasp is actually full of this drink, and his intention to lift the glass to his mouth to drink. The discovery of mirror neurons enables us, on the other hand, to say that observation of an action causes the activation of the same neural circuit in charge of controlling its execution; observation of the action therefore induces automatic simulation of that same action in the observer, and, through this, his understanding. As D. Stern wrote, "our nervous systems have been constructed to be captured by the nervous systems of others, to such an extent that we can feel and have the experiences of others as if we were in their skin and in the same way as if we were within our own skin" (Stern, 2004, p. 76).

We can therefore understand the meaning of others' behaviour precisely because our brain has the possibility and capacity to create models of this behaviour in the same immediate, automatic way in which it creates models of our own. The final result of this modelling process puts us in a position to understand and predict the consequences of others' action just as it enables us to understand and predict our own behaviour. The 
mechanism the two processes of understanding are based on is the same.

An important consequence of this illustration is that it is impossible for us to know other people and in particular what they do independently from ourselves without relating to our body and its movements. "Another's reality" cannot be known as such, but only in close relation to the subject perceiving, observing and interacting with it. From this point of view an interesting hypothesis was put forward by Gallese, one of the members of the Parma team to whom we owe the discovery of mirror neurons, who predicted "the existence of 'somatosensory' mirror neurons that might contribute to giving us the capacity to identify the different body parts of others, basing them on the same parts of our own body" (Gallese, 2003, p. 39).

This hypothesis was actually confirmed by the results of experiments carried out by Gallese himself in collaboration with other colleagues (Keysers et al. 2004), which showed that the experience of being touched on a part of one's body determines activation of the same neural circuit as that activated when we observe someone else being touched on the same part of the body. The cortical region involved is the SII/PV area, located in the parietal operculum, commonly considered an exclusively tactile area. The same cortical region is therefore activated both when we experience in first person a tactile sensation located in a part of our body, and when we witness a similar sensory feeling experienced by someone else.

These results lead us to consider subjectivity as a process that develops within the sphere of inter-subjectivity and in which the relationship with another is constitutive and fundamental. As Bakhtin writes in his book Estetika slovesnogo tvorčestva (Aesthetics of literary creation), which appeared in 1979, and was published in Italian under the title L'autore e l'eroe, "among the values of the internal world, there is no value that aesthetically expresses my body and my soul and their organic artistic unity in the integral man; my body and soul are not built up within the horizon of my own activity, so my horizon cannot calmly close itself up and surround me like an outline of myself endowed with value. [...] In all aesthetic forms the organising force is made up of the other's category of values and our relationship with the other, one that is enriched by the excess of value my view has of the other and that enables transgredient fulfilment" (Bakhtin, 1988, pp. 169-170).

Therefore, as Bakhtin also emphasises in his Problems of Dostoievski's Poetics, each individual that has existed in time is, and cannot but be, a fundamentally open system, so that the relationship with another is in itself a constitutive element of his being: "Not the analysis of consciousness in the form of a single, unitary I, but analysis of the interaction of many consciousnesses endowed with equal rights and full value. A single consciousness lacks self-sufficiency and cannot exist. I become aware of myself and become myself only by revealing myself to another, through another and by another. The most important acts that constitute selfawareness are determined by the relationship with another's consciousness (with the you). Detachment, disunity, withdrawing into one's shell as the principal cause of loss of self. Not what happens inside, but what happens at the border of one's own and another's consciousness, on the threshold. And everything that is internal is not self-sufficient but is addressed outwards and becomes a dialogue: each internal experience reaches the border, meets others and in this encounter full of tension lies all its substance. It is a higher degree of sociality (neither external, nor concerning things, nor internal). In this Dostoievski opposes all decadent and idealist (individualist) culture and the culture of radical, hopeless solitude. He asserts the impossibility of solitude, the illusory nature of solitude. The existence of man (both external and internal) is very deep communication. To be means to communicate. Absolute death (not being) is the impossibility to be heard, recognised and remembered. To be means to be for others and, through others, for the self. Man does not have a sovereign internal territory, but is always and ever at the edge, and, looking into himself, he looks into the eyes of another and with the eyes of another" (Bakhtin, 1988, pp. 323-324).

The relationship of otherness is therefore inherent in the I, in that it is discovered within the subject, and is itself a dialogue, an I/other relation, the identity of which is formed in the border space, in the intermediate world between the self and another and between the self and external reality as a whole.

\section{Temporal intelligence}

What we call "temporal intelligence" is generally connected with the traditional linear conception of history, hinged on the idea of time as X god that symbolised the mechanical measurement of time, which led to time being perceived as flowing in a single direction, from past to future, following the rhythms of life and divided into a "before" and an "after", all in all homogeneous.

From the classical tradition, however, we inherited other conceptions of time, more suitable to the enhancement of design and the capacities it requires. First of all,

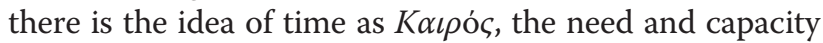
to seize opportunities that arise on the scene and rapidly fade away if we do not know how to grasp them. A concept of time, therefore, that presupposes the ability to find and maintain the right distance between, on the one hand, thought and action and, on the other, reality, for innovation and transformation to be able to take place. 
For this purpose the terms involved in the relation must not be too close, in order to avoid thought and action being overthrown by the course of events and effectuality reaching maturity and fulfilment, nor, however, too distant, to avoid them ending up losing contact with the "potential of the situation" and leaving the field of possibilities that arise, thus risking not being ready to

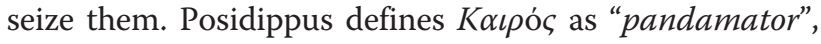
namely he who prevails over everything: he is on tip-toe, has double wings, holds a razor in his right hand, with his hair over his face and his neck bare. These are the features Posidippus picked out in Lysippos' statue, which translated into successful iconographic terms the idea of the right moment that has to be grasped as soon as it comes to us, otherwise it will be impossible to grasp that same impossibility to grasp the propitious moment that has irremediably passed which in Lysippean iconography translates into Kaı ó, , with no hair as a handhold. In The Nicomachean Ethics (1096a 27) Kaıpós is the definition of the good of time precisely because "action

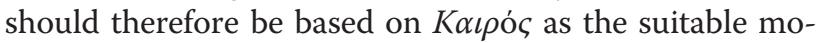
ment, i.e. should grasp the right time when it is ripe and decide upon action".

To speak of "suitable, right time" means to refer to the effort and objective of gaining advantage from circumstances and occasions, i.e. it indicates the patience of waiting for the situation to evolve so as to seize favourable developments and be able to find all the opportunities that might arise in circumstances as they develop, with the purpose of gaining advantage from them. The term Kaıpó

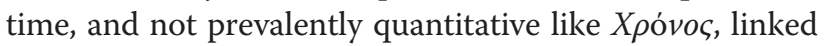
with the conviction that for each thing a moment of completeness and fulfilment exists. It describes the optimal moment for each thing, the culminating point but above all the decisional space for an action that aims to succeed and therefore achieve its own telos.

But there is a possible alternative etymological origin of this idea of time that makes its distinctive traits emerge with greater efficacy. This is the idea of time as

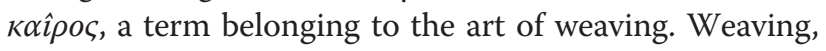
time and fate were ideas that were often linked. An opening in the weft of fate might mean a gap in time, an eternal moment in which the design becomes more compact or relaxed: the weaver pushes the shuttle and bobbin through the opening in the threads of the weft at the critical moment, the right moment, for the gap in the weft only has limited time and the shuttle must be pushed while the gap is open.

Finally, again in the ancient world, in Greek philosophy in particular, there is a clear reference to another type of "temporal intelligence", based on a conception of time not consisting just of the capacity to mark out 'becoming' and seize opportunities but also on a sense of permanency and continuity that has a dual nature: the experiential nature of each single individual and the one we define nowadays as phylogenetic-species specific, which underlies the simultaneous feeling of individual uniqueness/privacy and universality. This conception of time hinges on the awareness that personal memory is solidly attached to the "collective memory" at the foundations of culture and re-proposes it, confirming and moulding it continually. The connection and interaction between this dimension of collective memory and the overall experience of each single person in the "here" and "now" in which they live are characterised and marked by the time of permanency, Ali $\dot{v} v$, which guarantees continuity between the different generations - what Arthur Lovejoy calls "the great chain of being" (Lovejoy, 1966). The contents archived in the individual mind events, facts, concepts and capacities - are precisely for this reason something more than the representation of a single, unique personality. They are also the crucial point of transmission of culture. Time as Alív is the active subject of this transmission, the base on which it rests and what makes each one of us a "transmission belt" of the inheritance of the past and the centre of projects of the history of the future.

But besides this eternal, unchanging Alív that is beyond time, there is another type, however, that becomes real in the world and enables "duration" - the birth, growth and then the end of each process of manifestation. On the temporal plane this cannot but be expressed as past, present and future, while at the symbolic level it becomes $A \mathfrak{l} \omega v$ that is initially the boy, then the adult and finally the old man. These are indeed the bestknown portrayals of the god $A \mathfrak{l} \omega \dot{\omega} v$, represented as a boy not only in rituals but even in the first Hellenic conception (Heraclitus, then Euripides).

The Aí $\dot{v}$ symbol expressed through the three fundamental ages that mark out the moments of a cosmic cycle is found again, significantly, in the House of $A \mathfrak{l} \omega v$ mosaic in Antioch, dating back to halfway through the Third Century A.D. This mosaic stands out for a particular detail: it depicts a symposium or triclinium where four male figures are shown lying on klinai. The figure on the left, a mature though not elderly man with a beard and moustache, wearing a garland of leaves and holding the Zodiac wheel in his right hand, rests on another kline, of which only the headpiece can be seen. He is looking towards his left at three individuals facing forwards and lying on a kline. Next to the figure on the left lying on the bed of which only the headpiece is seen, the word aion appears, while next to the three figures on the couch facing forwards three words can be read respectively: mellon (future), enestos (present) and finally parochemenos (past). Moreover, between the bed-head on the left and the trapezion (table) another inscription 
appears - chronoi (times) - which presumably refers to the three male figures seen from the front. The strangest aspect of the portrayal in mosaic that naturally makes the interpretation more interesting is finding aion and chronoi together. This cannot but bring to mind - also for chronological issues - the title of Plotinus' Enneads, Treatise III 7, On Eternity and Time, in which he attributes the temporality of duration to the Soul, this hypostasis being more in contact with the sensible world, and atemporal eternity to the nous. Significantly Plotinus writes (Enn., III 7 7-8) that to investigate time one must descend (katabateon) from eternity. At the chronological level of Plotinus and probably also of the Antioch mosaic the meaning of aion was widely stabilised: in effect, by this term was meant, on the one hand, eternity as opposed to the durative aspects of temporality, and on the other, as perhaps was the case of the mosaic in question, eternity defined in the duration of time, past, present and future.

The presence of these mixed, hybrid forms authorises and may give credit to a hypothesis of some interest, namely they might constitute the necessary reference base for conceptually framing those particular forms of experience that, though fitting into determinate time and a specific phase of becoming, manage to cross the barriers of the immediate and the present, to fit into that atemporal dimension that is determined precisely by the capacity to place oneself outside the incessant flow of instants, so as to experiment a sort of suspension of the rhythm of time. It is exactly this that gives emotions that kind of imprint of eternity thanks to which they open a breach in time and endure, managing to pass from one generation to another.

We are obviously referring to that type of perception that constitutes the opportunity, motivation and kind of experimentation to rise to the dimension of art, something that can be likened to an "interruption in time", an experience of "absorbed, intransitive attention" - a sort of "out of time within time", a breath of eternity in the experience of life. This type of perception presupposes the capacity to isolate a single instant (one in which a significant experience takes place) and "condense" past, present and future in it, subtracting it from the rhythm of time and thus introducing into the latter a break which, as Giorgio Agamben writes "seems to introduce a split or halt in this eternal flow" (Agamben, 1984, pp. 150-151).

Reference to this rich, articulate analysis of time is more important and topical than ever, given that, as we said at the beginning, a cultural perspective is still in force and dominant that proceeds according to the logic of immediate, brief time and follows the strict dynamics

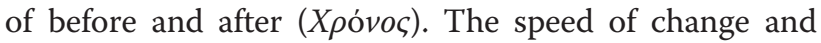
innovation nowadays, however, requires more and more reference to time as the capacity to seize opportunities, establishing the right distance between thought and ac-

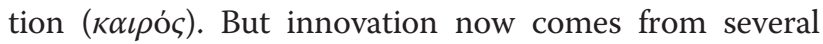
different sources and the growing success of the open, widespread idea of governance, opposing the oligarchic, hierarchical one of government, involves reference to several subjects, both institutional and social, so that the idea of synchronisation and "weaving" between different impulses, wishes and decisional levels is decisive. In this

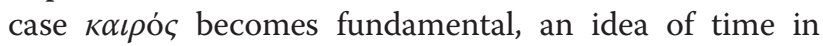
greater harmony with governance, which is a dynamic process and a normative, regulatory idea. The impossibility to define specific confines within it makes games devoid of well-defined lines of demarcation and actors that are not all defined and certified beforehand. The consequence is the need to keep the co-presence of what is systemic and extrasystemic, structural and extrastructural, and to know how to "weave them together", since the extrasystemic/extrastructural components function more and more as a reserve of energy and creativity from which to draw to give new impulse, dynamism and creativity to the system. Subsidiarity relations, partnerships, systematic negotiations and contracted policies should be set up, but cannot be shut up in local government politics. Strategic plans, not by chance, are relaunched onto several generations and open up the future; they have necessarily to take into account not just the balance between local and opening up to the global, but also opening up to time as duration, to $A \mathfrak{l} \omega \dot{\omega} v$ in all its extension and duration.

\section{"Double vision"}

What we have called "contextually bound flexibility", made up, as we have seen, of "roots", "wings", "inhabiting" strongly rooted in the context one belongs to, with its territorial, historic, cultural and social specificities, and a vocation for migration, for launching oneself into a different space from that of our everyday experience, needs, in order to develop adequately, to be based on a type of vision of which Giacomo Leopardi left us an exemplary analysis: "To the sensitive, imaginative man, living, as I did for a long period, continuously feeling and imagining, the world and objects are in a certain sense double. Through his eyes he will see a tower, the countryside; with his ears he will hear the sound of a bell; at the same time, with his imagination he will see a different tower and different countryside, and will hear a different sound. All that is beautiful and pleasant in things lies in this second kind of object. Sad is that life (and it frequently is, too) that does not see or hear or feel, except for simple objects, just those of which his eyes, ears and other senses receive sensations" (Leopardi 1997, 30 November).

A secondary image thus unfolds, capable of opening up deeper and wider horizons and stimulating the mind's 
creativity and capacity to "see and think differently" compared with the usual, dominant patterns.

On the grounds of this profound intuition of Leopardi's, it is not just acceptable but dutiful to conclude that the environment in which we live inexorably decays and grows ugly if we remain prisoners of a gaze that "sees without feeling" and, devoid as it is of any ability to imagine, levels out reality as it appears to lazy eyes and a perceptive structure incapable of expressing and producing that "second image" of which Leopardi speaks (Leopardi 1997).

Leopardi's deep insight, which also clarifies the sense of the quotation from Wittgenstein at the beginning of this article, has received interesting confirmation in the model of information processing in three stages, each of which transforms and enriches the previous one, which contemporary neuroscientists have developed as regards visual perception, in the wake of David Marr's pioneering work (Marr 1982).

These stages have been summarised as follows by Erik K. Kandel: "The first stage, which begins in the retina, is the low-level visual processing studied by Kuffler. This stage establishes the features of a particular visual scene, singling out the position of an object in space and identifying its colour.

The second stage, which begins in the primary cortex, is the intermediate level of visual processing described by Hubel and Wiesel and by Zeki. It assembles simple linear segments, each aligned in a specific direction, obtaining outlines that define the borders of an image, and builds up coherent perception of the shape of an object. This process is called outline integration. At the same time the intermediate level of sight separates the object from the background in a process called surface segmentation. Lowand intermediate-level processing together identify as figures the areas of the image that are linked with an object and as background the areas that are not [...].

The third stage, high-level visual processing, which spreads along the primary visual cortex to the lower temporal cortex, establishes categories and meanings. Here the brain integrates the visual information with relevant information coming from a variety of other sources, and enables us to recognise specific objects, faces and scenes" (Kandel, 2012).

Of these two phases, that of intermediate-level visual processing is considered particularly demanding for it requires that the primary visual cortex determine which segments belong to a single object and which are components of other objects in the context of an overall visual scene, composed of hundreds or even thousands of segments of lines.

Here the decisive importance of the outline as a line of demarcation can be seen. Studies on the methods of organisation of the visual world of all species able to focus light to form images actually show that these methods must, however, be characterised by the presence of segregated figures, clearly distinct against the background. Given the properties of light, there are few ways of obtaining this. One, a very general way, is to obtain margins or edges where physical stimulation reveals differences. The problem is of course that in many circumstances these physical variations may not be very clear, if not indistinct, or may just be present in parts (think of an animal moving among thick foliage). And so, by means of natural selection, interpolation mechanisms have been perfected, which, using quite simple rules based on the statistical regularities of the environment (similarity in colour, clarity and texture, continuity of direction, common movement of parts, etc.), extract edges and lines of demarcation for the use and consumption of the animal that needs them.

This is exactly why "our brain cells are very good at reading lines and profiles as edges. The brain integrates simple lines to form the edges differentiating a figure from the background. Whenever our eyes are open, orientation cells in the primary visual cortex are constructing the elements of the picture of the scene before us. Moreover, the primary visual cortex uses the inhibitory regions of the perceptive fields of those neurons to accentuate the border lines of the image" (Kandel, 2012 pp. 272-273).

This tendency to pick out margins and lines of demarcation is so strong that living organisms tend to see such lines even where they do not physically exist, like in the famous figures of Kanizsa. We are referring to situations in which we find ourselves faced with an unusual figure that in actual fact is not there, even though it is seen, and "seeing the invisible" is the tangible expression and proof of the fact that we need a border that separates and distinguishes figure and background; we need it to such a degree that even if this border is not physically there, we are steered towards perceiving it just the same. In these cases, then, the border takes shape as an edge, a line of demarcation that enables the visual scene to be divided up according to the rules of the figure/background game.

Researchers studying the brain have discovered some thirty centres that, as well as the primary visual cortex, continue the task of analysing and isolating, or segregating, information on shape, colour, movement and depth. The information coming from all these specialised areas is segregated and conveyed separately to the higher cognitive regions of the brain, including the prefrontal cortex, where it is finally coordinated in unitary, identifiable perception.

The low- and intermediate-level processing stages are carried out together and mostly follow a bottom-up course based on principles studied by the Gestaltists, such as the proximity of the linear segments that form 
the outline of an object, similarity in colour, dimension and orientation, and good continuity, according to which in a figure the segments of a line will usually be oriented and grouped together in such a way that the edges continue in the "smoothest" way possible.

High-level visual processing is top-down. It produces inferences and controls hypotheses comparing them with the visual images remembered, of which it has previously had experience, and thus leads to aware visual perception and interpretation of meaning, which is nevertheless not perfect and may lead to errors.

Top-down re-evaluation operates following four principles:

a) Neglecting the details that are not behaviourally relevant in a given context;

b) Looking for their constancy;

c) Trying to draw out the essential, constant features of objects, people and landscapes;

d) And, finally, something particularly important, comparing the present image with images encountered in the past.

These biological results confirm that sight is not simply a window on the world, but really is the result of active processing by the brain. As the cognitive psychologist Chris Frith writes: "What I perceive are not the rough, ambiguous suggestions that reach my eyes, ears and fingers from the outside world. I perceive something much richer, an image that combines all these rough signals with an enormous quantity of past experiences. Our perception of the world is a fantasy that coincides with reality" (Frith, 2009, p. 167).

All these findings seem to confirm the fact that creative work requires priority reference to the original sphere of the possible, with its almost unlimited opportunities, from which a "top-down" route develops, which leads, following a tenacious, constant effort of selection and narrowing down, to the system of ties dictated and imposed by adherence to effectuality, namely to the real which appears to us "here" and "now".

\section{Deduction, induction and abduction}

These results reinforce the doubts and bewilderment already pointed out in the first paragraph as regards the idea that we may speak of a "mechanism of discovery", and also therefore of project-oriented processing based entirely on an inductive process.

Induction, as is known, gives rise to "Hume's problem", which Kant also came up against, and from which the "Copernican revolution" sprang, carried out by him in The Critique of Pure Reason. This consists of trying to understand how we move from a multiplicity of observations to a theory that enables the behaviour of nature to be envisaged. Is the inductive procedure correct and scientifically reliable, Hume wondered, when it authorises us to pass from many specific cases to one general statement? Hume's typical example was this: how can we be certain that the Sun will rise tomorrow based on the fact that each day past experience has taught us that the Sun does rise? Is there a reason why the future must necessarily resemble the past? The answer he provided was sceptical: induction is not a reliable instrument for seeking truth, in that it is based on an unjust transformation of temporal succession (post hoc) into a causal link (propter hoc). Nevertheless, man is led to "believe" in induction (beliefs of the type "tomorrow the Sun will rise") because he is guided by habit. Something I have very often seen happen leads me to believe that I will see it happen again.

Bertrand Russell presented a particularly witty, pungent variation of the problem. Think of an American turkey that is fed every day. At each meal its conviction is consolidated that one of the general rules of life is that it will be fed daily by friendly members of the human race who think only of its welfare. Its faith gains strength gradually as the number of meals administered to it grows, and each day the idea that it is in a friendly environment is corroborated. As the days pass and its death grows nearer and nearer, the turkey feels more and more secure. Its sense of security, therefore, reaches a maximum just at the moment when risk becomes greatest. This is the trouble and the paradoxical aspect of induction: learning backwards, trusting in the uniformity of the course of nature and experience, and in the impossibility of a radical change. Then, however, in view of Thanksgiving, the turkey experiences something totally unexpected: it is strangled in order to be cooked.

Charles Sanders Peirce (1839-1914) suggested a "third way" compared with the two processes and instruments for thinking that we have so far taken into consideration. Particularly interested in understanding what scientific method consisted of and its features, he tried to reconstruct the way the scientist reasons in his scientific work. To this end he developed the philosophy of science in his theory on inference, in which by inference we should understand not a series of mental processes - in actual fact, from the psychological point of view we may arrive at a theory from the most unexpected ways - but the set of types of scientific reasoning and kinds of justification that can be offered for them. On this matter he distinguished three different fundamental ways of reasoning: deduction, induction and retroduction (usually translated by abduction). Deduction is reasoning that, if used correctly, cannot lead from true premises to a false conclusion; given the truth of the premises, the truth of the conclusion must necessarily follow. The need for deductive reasoning depends on the fact that it is not exposed to possible empirical confutation. Deductive reasoning, logical 
and mathematical reasoning, is valid in every possible universe. Induction, on the other hand, is that kind of reasoning by which one concludes that facts similar to those observed are true in cases not examined. Thus, for example, from the fact that all swans observed up to now have proved to be white, it appears legitimate to conclude that other swans will also be white. In Peirce's opinion, abduction (or retroduction or hypothetic reasoning) should be taken into consideration as well as deduction and induction. The differences existing between induction and abduction are basically two: in the first place, in induction we conclude, as stated, that facts similar to those observed are true in cases not examined, while in hypothetic reasoning or abduction we reach the conclusion that a fact exists that is completely different from any other observed up to now; in the second place we can see that while induction classifies, abduction explains. The pattern of reasoning by abduction is as follows:

1. We observe $C$, a surprising fact.

2. But if A were true, then $C$ would be natural.

3. There is, therefore, reason to suspect that $\mathrm{A}$ is true.

What is thus being maintained is that a certain conjecture (or hypothesis), i.e. that A is true, is worth taking into consideration. We therefore see that abduction is the fruit of the scientist's inventive, creative moment, of the fortunate instant of scientific inventiveness that formulates generalised explicative hypotheses, which, if confirmed, become scientific laws (though still able to be corrected and substituted) while, if falsified, are rejected. And it is indeed abduction that makes scientific and technological thought progress, conveyed forward on the one hand by the progressive incorporation of new, unsuspected facts that therefore stimulate the invention of new hypotheses able to explain them and, on the other, by an axiomatic unification of laws, implemented by what are called the great simple ideas.

Henri Poincaré had already ingeniously understood this when over a century ago he observed on the subject of the scientist's behaviour, with originality and a capacity for foresight that still do not cease to amaze us today, that when the scientist finds himself faced with data and observations that constitute the material of his work, he should "not acknowledge so much the similarities and differences, as, rather, single out the affinities hidden under apparent discrepancies. The particular rules seem at first sight discordant but, on looking closer, we realise that they are usually similar; although presenting material differences, they resemble each other in the shape and order of their parts. Considering them from this standpoint, we will see them extend and tend to become all-embracing. And it is this that gives value to certain facts that link up to complete a whole, showing how it is the faithful image of other sets already known. I do not want to go further with this; these few words are enough to show that the man of science does not choose the facts he must observe by chance [...]. He tends rather to concentrate a lot of experience and much thought in a slim space, and this is why a small physics book contains so much past experience and a number of possible experiences a thousand times greater, the results of which we already know" (Poincaré, 1997, pp. 14-15).

The man of science does not, therefore, proceed by piling up and hoarding facts and data or acting on the sum of them, but by intersection and interlocking, finding bridges and analogies under the diversities that appear that are not detectable by an untrained, nonexpert eye. In this way he manages to establish links, transfer and superimpose so that he is able to considerably reduce the volume of experiences, both effectively achieved and simply possible, which he has available.

According to Peirce, however, though acknowledging the priority and specific function carried out by the process of hypothetic inference, we need to realise that abduction is closely connected with deduction and induction. It is so in the sense that, having to judge the acceptability of the hypothesis, it will be necessary for each true, plausible hypothesis to be such that consequences can be deduced from it which, in their turn, may be inductively tested, namely by experiments. Furthermore, in his opinion, this dependency does not have a one-sided nature, since he considers induction above all a method for testing conclusions, and he believes these conclusions are always suggested, in the first place, by hypothetic inference. With induction consequences are generalised and tested that can be deduced from a given hypothesis; thus, the reciprocal dependency of these two forms of inference and their common dependency on deduction prove to be equally clear. In other words, we cover the world and the myriad facts composing it with hypotheses or conjectures of a general nature in order to understand, foresee and manipulate them, and from these we can deduce unusual propositions that, if verified, will confirm those hypotheses, which will then pass on to the rank of laws, though always revisable.

So here we have reached, in conclusion and on the grounds this time of a path of discussion, the idea that project-oriented capacity - precisely because it requires the possession of, and capacity to correctly manage, the entire register of "instruments for thinking" that we have available, given that as well as deduction, induction and abduction it presupposes the current use also of analysis, abstraction and analogy - may only be developed, as already mentioned, on the basis of an itinerary of deep, complete enhancement of the designer as a person and of his specific skills, capacities and knowledge. It is in this sense that the idea concisely expressed in the title of 
this paper should be understood, according to which "to design is to design oneself". Between the two moments of personal growth and designing "tangled recursivity" exists, of the type Hofstadter speaks of in his book Gödel, Escher, Bach, basing it on the following conjecture: "recursive systems sufficiently complex to possess the strength necessary to evade all pre-established patterns might exist. And is this not perhaps one of the properties that define intelligence? Instead of simply considering programmes as composed of recursive procedures able to call up themselves, why not make a real effort and invent programmes able to modify themselves: programmes able to act on programmes, extend, improve and repair them, and so on?" (Hofstadter, 1994, p. 165).

Read in this key, between designing and designing oneself a relation is configured by means of which the first process is developed and becomes manifest through the second, which, in turn, grows and progresses thanks also to the internal resources acquired and enriched by commitment to the exercise of the art of designing however understood.

\section{Competing interests}

The author has no competing interests and has approved the final version of the manuscript.

Received: 1 November 2013 Accepted: 28 February 2014

Published: 16 May 2014

\section{References}

Agamben G (1984) L'uomo senza contenuto. Quodlibet, Macerata Aristotele (2012b) In: Natali C (ed) Etica Nicomachea. Laterza, Rome-Bari Bakhtin M (1988) L'autore e l'eroe. Teoria letteraria e scienze umane. Einaudi, Turin Calvino I (2002) Lezioni americane. Sei proposte per il prossimo millennio, Mondadori, Milan, 2002, Eng. trans. Six Memos for the Next Millennium. The Charles Eliot Norton Lectures (2008). Vintage International, Vintage Books, New York

Ferretti F (2009) Alle origini del linguaggio umano. II punto di vista evoluzionistico, Laterza, Rome-Bari. This work refers in particular to the research of Spreng and collaborators, published in N. Spreng, R.A. Mar, A.S.N. Kim, The common neural basis of autobiographical memory, prospection, navigation, theory of mind, and the default mode: A quantitative meta-analysis. J Cogn Neurosci 21(3):489-510

Frith C (2009) Inventare la mente. Come il cervello crea la nostra vita mentale. Raffaello Cortina, Milan

Gallese V (2003) Neuroscienza delle relazioni sociali. In: Ferretti F (ed) La mente degli altri. Prospettive teoriche sull'autismo. Editori Riuniti, Rome

Hofstadter D (1994) Gödel, Escher, Bach: un'eterna ghirlanda brillante. Adelphi, Milan

Kandel EK (2012) L'età dell'inconscio. Arte, mente e cervello dalla Grande Vienna ai nostri giorni. Raffaello Cortina, Milan

Keysers C, Wickers B, Gazzola V, Anton J-L, Fogassi L, Gallese V (2004) A Touching Sight: SII/PV Activation during the Observation and Experience of Touch. Neuron 42(22): 1-20

Lacan J (1974b) In: Scritti Contri G (ed) Lo stadio dello specchio come formatore della funzione dellio, Vol. Ith edn. Einaudi, Torino

Leopardi G (1997b) In: Damiani R (ed) Leopardi 1828, 30 November. Zibaldone, Mondadori, Milan

Lovejoy A (1966) La grande catena dell'essere. Feltrinelli, Milan

Marr D (1982) Vision: A computational Investigation into the Human Representation and Processing of Visual Information. W.H. Freeman \& Company, New York

Merleau-Ponty M (1965) In: Bonomi A (ed) La fenomenologia della percezione. II Saggiatore, Milan

Musil R (1957) L'uomo senza qualità. Einaudi, Turin
Pirsig RM (1981) Lo Zen e l'arte della manutenzione della motocicletta. Adelphi, Milan

Poincaré JH (1989) Pensieri ultimi, in Id. In: Boniolo G (ed) Opere epistemologiche. Piovani, Abano, Terme

Poincaré JH (1997) In: Bartocci C (ed) Scienza e metodo. Turin

Postman N (1983) Technopoly. La resa della cultura alla tecnologia. Bollati Boringhieri, Turin

Rizzolatti G, Sinigaglia C (2006) So quel che fai. II cervello che agisce e i neuroni specchio. Raffaello Cortina, Milan

Stern DDN (2004) The present moment in psychotherapy and everyday life. W.W. Norton \& Co., New York

Taleb NN (2008) II Cigno nero. Come l'improbabile governa la nostra vita, II. Saggiatore, Milan

Wittgenstein L (2002) In: De Palma A (ed) The big Typescript. Einaudi, Turin

\section{doi:10.1186/2195-2701-1-8}

Cite this article as: Tagliagambe: To design is to design oneself.

City, Territory and Architecture 2014 1:8.

\section{Submit your manuscript to a SpringerOpen ${ }^{\circ}$ journal and benefit from:}

- Convenient online submission

- Rigorous peer review

- Immediate publication on acceptance

- Open access: articles freely available online

- High visibility within the field

- Retaining the copyright to your article

Submit your next manuscript at $>$ springeropen.com 